\title{
Can reducing sitting time in the university setting improve the cardiometabolic health of college students?
}

This article was published in the following Dove Press journal: Diabetes, Metabolic Syndrome and Obesity:Targets and Therapy

\author{
Karrie M Butler' \\ Joyce S Ramos ${ }^{2}$ \\ Christina A Buchanan' \\ Lance C Dalleck ${ }^{1,2}$
}

'Department of Recreation, Exercise, and Sport Science, Western State Colorado University, Gunnison, CO, USA; ${ }^{2}$ SHAPE Research Centre, Exercise Science and Clinical Exercise Physiology, College of Nursing and Health Sciences, Flinders University, Adelaide, SA, Australia
Correspondence: Lance C Dalleck Department of Recreation, Exercise, and Sport Science, Western State Colorado University, 600 North Adams Street, Gunnison, CO 8I23I, USA

$\mathrm{Tel}+19709437132$

Fax+l 9709437125

Email Idalleck@western.edu
Purpose: The high prevalence of metabolic syndrome (MetS), prediabetes, and increased risk of cardiovascular diseases linked with prolonged sitting has created a need to identify options to limit sedentary behaviors. A potentially simple approach to achieve this goal in the university setting is to provide students the option to stand during courses rather than sit. The purpose of the present study was to examine the effects of standing in the college classroom setting on cardiometabolic risk factors in a cohort of college students.

Patients and methods: Healthy college students $(n=21)$ who attended at least two courses per week (a minimum of 5 hours) in a specified university building with standing desks participated in a 7-week intervention that was divided into three phases: 3 weeks of standing, 1 week of washout (sitting), and 3 weeks of sitting. The participants (mean \pm SD: age, height, weight, body mass index, and waist-to-hip ratio were $22.7 \pm 6.4$ years, $174.3 \pm 10.0 \mathrm{~cm}, 70.6 \pm 14.3 \mathrm{~kg}, 23.0 \pm 3.0$ $\mathrm{kg} / \mathrm{m}^{2}$, and $0.76 \pm 0.05$, respectively) were randomly assigned to the phase of intervention of which they should start (sitting or standing), and all participants engaged in sitting during the washout phase. Cardiometabolic risk factors and metabolic equivalents (METs) were measured at baseline and weekly throughout the intervention.

Results: Paired $t$-tests revealed significant differences $(P<0.05)$ in all cardiometabolic risk factors between the 3 weeks of sitting and 3 weeks of standing time blocks. Moreover, MetS $z$-score was significantly improved $(P<0.05)$ during the 3 weeks of standing $(-5.91 \pm 2.70)$ vs 3 weeks of sitting $(-5.25 \pm 2.69)$. The METs were significantly higher $(P<0.05)$ during standing $(1.47 \pm 0.09)$ than during sitting $(1.02 \pm 0.07)$. Although there was considerable interindividual variability in the $\Delta$ MetS $z$-score response, there was a $100 \%(21 / 21)$ incidence of a favorable change (ie, responders) in MetS $z$-score response.

Conclusion: A standing desk in the classroom paradigm was found to significantly improve cardiometabolic health throughout a short 3 weeks time span. Increasing standing time in the classroom, and therefore lessening weekly sedentary behavior, could be a potential wide-scale, effective strategy for primordial prevention of cardiometabolic diseases.

Keywords: inactivity physiology, primordial prevention, sedentary behavior

\section{Introduction}

Cardiovascular disease (CVD) is widespread in the US, but it is largely preventable with changes in lifestyle. The most prominent risk factors for CVD are dyslipidemia, physical inactivity, and obesity. ${ }^{1}$ Within a university setting, college students are a population perceived to be relatively healthy and have low risk for such diseases due to their younger age and being relatively active, but this may be a misconception. Unfortunately, many students are unaware of the presence of these risk factors because of 
infrequent testing and appearing to have a healthier lifestyle; however, much of their time is spent sitting during courses, studying, and exhibiting sedentary behaviors as a whole due to them being students. For example, Dalleck and Kjelland ${ }^{2}$ found that $6.8 \%$ of college students had metabolic syndrome (MetS), 13.0\% had two risk factors for MetS, and $42.5 \%$ of the sample exhibited one risk factor for MetS. For an average adult, the recommended guideline for physical activity (PA) is 2.5 hours of moderate-intensity aerobics per week. Individuals can meet the recommended PA guidelines, but still live a less-than-ideal lifestyle. Sedentary individuals who follow the weekly recommendations for PA, but are sedentary for great lengths throughout the day, are at a higher risk for cardiometabolic diseases relative to their less sedentary counterparts who exercise the same amount. ${ }^{3}$ Unfortunately, meeting the PA guidelines alone cannot overcome the negative effects of high amounts of daily sedentary behavior.

With the high prevalence of MetS, dyslipidemia, and prediabetes and an increase in the risk of CVD due to prolonged sedentary behavior, there is a need to include options to limit these sedentary behaviors, especially in a university setting. A simple approach to achieve this goal is to provide students the option to stand during courses rather than sit. A recent publication on the feasibility of standing desks in a university-based classroom found that $95 \%$ of students preferred the option to stand. ${ }^{4}$ Therefore, standing desk could be a simple way to lessen the negative cardiometabolic effects of increased sedentary behavior. To our knowledge, there have been no prior studies investigating the effectiveness of standing desks in the university setting. The purpose of the present study was to assess the effectiveness of a standing desk university classroom model for attenuating cardiometabolic risk in a cohort of college students. It was hypothesized that incorporating standing desks into the university classroom setting will improve the cardiometabolic risk factor profile of college students.

\section{Materials and methods}

\section{Participants}

Participants $(n=21)$ were recruited through poster advertisement, word-of-mouth, and email between November 2017 and January 2018. The characteristics of participants who completed the study $(n=21)$ are shown in Table 1 . Each participant was asked to read and sign an informed consent form outlining the study procedures and goals of the study, and agreeing that her/his participation was voluntary. Additionally, each participant was informed verbally and in writing that the consent could be withdrawn at any time without any consequence. Inclusion criteria were: 1) $\geq 18$ years old and
Table I Participant characteristics (values are mean \pm SD and \%)

\begin{tabular}{|l|l|l|l|}
\hline Parameter & $\begin{array}{l}\text { Combined } \\
(\mathbf{n = 2 1})\end{array}$ & $\begin{array}{l}\text { Males } \\
(\mathbf{n}=\mathbf{I 3})\end{array}$ & $\begin{array}{l}\text { Females } \\
(\mathbf{n = 8})\end{array}$ \\
\hline Age (years) & $22.7 \pm 6.4$ & $21.2 \pm 1.2$ & $25.3 \pm 10.2$ \\
Height (cm) & $174.3 \pm 10.0$ & $180.0 \pm 7.6$ & $165.2 \pm 5.7$ \\
Weight (kg) & $70.6 \pm 14.3$ & $78.8 \pm 11.3$ & $57.2 \pm 5.9$ \\
BMI (kg/m $\left.{ }^{2}\right)$ & $23.0 \pm 3.0$ & $24.3 \pm 3.0$ & $20.9 \pm 1.3$ \\
Waist (cm) & $74.4 \pm 6.9$ & $78.5 \pm 4.7$ & $67.8 \pm 4.3$ \\
Waist-to-hip ratio & $0.76 \pm 0.05$ & $0.79 \pm 0.03$ & $0.73 \pm 0.05$ \\
Ethnicity & & & \\
Caucasian (\%) & 71.4 & 53.8 & 100.0 \\
Black (\%) & 23.8 & 38.5 & 0.0 \\
Hispanic (\%) & 4.8 & 7.7 & 0.0 \\
\hline
\end{tabular}

Abbreviation: BMI, body mass index.

2) a college student at Western State Colorado University with at least two classes in a single, specified building on campus. Exclusion criteria were: 1) pregnancy and 2) an injury/medical condition that would prevent standing for an extended period of time. This study was approved by the Human Research Committee of Western State Colorado University (HRC2017-02-02R03).

\section{Experimental design}

This randomized, crossover trial was designed to evaluate the effects of increased standing time on various cardiometabolic markers, including fasting blood glucose, high-density lipoprotein (HDL) cholesterol, triglycerides, and SBP/DBP. Data were collected from January to March 2018. All participants were part of the intervention and served as their own controls. The intervention trial required standing during at least two different class periods per week, utilizing standing desks; this totaled to a minimum of 5 hours per week of standing for 3 weeks. The control trial required sitting in the same two classes for 3 weeks. The control and intervention trials were separated by a one-week washout period in which all participants were required to sit. The participants were randomized to the trial which they would start with first.

At baseline, measures were obtained for the following variables: age, height, weight, waist circumference, hip circumference, resting blood pressure, and fasting lipids/ blood glucose. Additionally, each participant also filled two survey questionnaires regarding PA and sedentary behavior: International Physical Activity Questionnaire and Sedentary Behavior Questionnaire. Each week throughout the study, with the exception of the washout week, fasting blood glucose and lipid profiles and resting blood pressure were measured. Further, on one occasion in the intervention, a metabolic cart was used to measure gas exchange data between standing and 
sitting for each participant to establish metabolic demands of the activities. A flow diagram of the experimental design outlining the timing of measurements throughout the duration of the study is presented in Figure 1.

\section{Procedures}

\section{Anthropometric measurements}

All anthropometric measurements were obtained using standardized guidelines. ${ }^{5}$ The participants were weighed to the nearest $0.1 \mathrm{~kg}$ on a medical-grade scale and measured for height to the nearest $0.5 \mathrm{~cm}$ using a stadiometer. Waist and hip circumference measurements were obtained using a cloth tape measure with a spring-loaded handle (Creative Health Products, Ann Arbor, MI, USA). For waist circumference, a horizontal measurement was taken at the narrowest point of the torso (below the xiphoid process and above the umbilicus). Hip circumference measures were obtained at the point where the buttocks extended the maximum when viewed from the

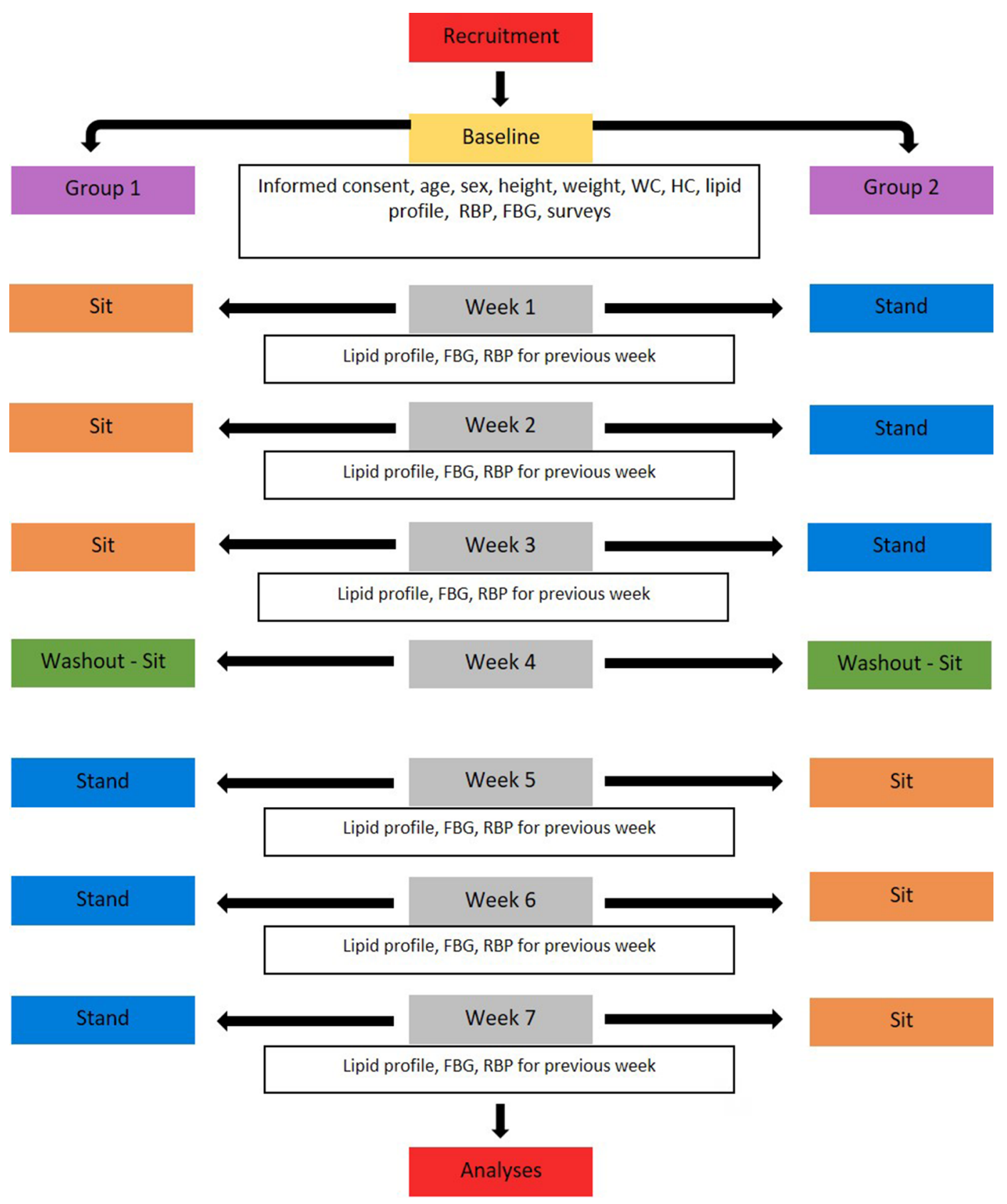

Figure I Experimental flowchart.

Abbreviations: FBG, fasting blood glucose; HC, hip circumference; RBP, resting SBP/DBP; WC, waist circumference. 
side. Measurements of each site (ie, waist and hip) were taken three times before averages were calculated and recorded. Waist-to-hip ratios were calculated using the averages.

\section{Fasting blood lipid and blood glucose measurement}

A fasting blood sample was collected and analyzed for the measurement of blood lipid profile and glucose. Participants' hands were washed with soap and rinsed thoroughly with water, then cleaned with alcohol swabs, and allowed to dry. Skin was punctured using lancets, and a fingerstick sample was collected into heparin-coated $40 \mu \mathrm{L}$ capillary tube. Blood was allowed to flow freely from the fingerstick into the capillary tube without milking of the finger. The samples were then dispensed immediately onto commercially available test cassettes for analysis in the Cholestech LDX System (Alere Inc., Waltham, MA, USA) according to strict standardized operating procedures. The Cholestech LDX system measured total cholesterol, HDL cholesterol, low-density lipoprotein cholesterol, triglycerides, and blood glucose in the fingerstick blood. A daily optics check was performed on the Cholestech LDX analyzer.

\section{Resting blood pressure measurement}

The procedures for measuring resting blood pressure outlined elsewhere were followed. ${ }^{5}$ Briefly, the participants were seated quietly for 5 minutes in a chair with a back support with feet on the floor and arm supported at the heart level. The left arm brachial artery blood pressure was measured using a sphygmomanometer in duplicate at 1-minute interval. The mean of the two measurements was reported for baseline and weekly values.

\section{MetS z-score}

A continuous risk score assessment scale (MetS $z$-score) was used previously to identify changes in MetS risk factors following an exercise intervention. ${ }^{6}$ The MetS severity was presented as sex-specific MetS $z$-score calculated using the following equations: $\left.{ }^{7} 1\right)$ MetS $z$-score men $=[(40-$ HDL-C $) / 8.9]+[(\mathrm{TG}-150 / 69)]+[(\mathrm{FG}-100) / 17.8]+[(\mathrm{WC}$ $-102) / 11.5]+[(\mathrm{MAP}-100) / 10.1] ; 2) \mathrm{MetS}_{z}$-score women $=[(50-$ HDL-C $) / 14.5]+[(\mathrm{TG}-150 / 69)]+[(\mathrm{FG}-100) / 17.8]+[(\mathrm{WC}$ $-88) / 12.5]+[(\mathrm{MAP}-100) / 10.1]$, where $\mathrm{FG}=$ fasting glucose, HDL-C = high-density lipoprotein cholesterol, MAP = mean arterial pressure, $\mathrm{TG}=$ triglycerides, and $\mathrm{WC}=$ waist circumference.

\section{Standing desk protocol}

The standing desks were located within the Department of Recreation, Exercise, and Sport Science at the local univer- sity. All participants used the standing desks for 3 weeks in two of their classes, totaling to a minimum of 5 hours per week. The participants were instructed not to lean on the desks or other stationary items while using the desk to avoid contamination of results during data collection. They were also encouraged not to stand outside of their requirements for this study nor did they keep a log. The participants were asked each week at the lipid profile test regarding whether they attended their classes, stood in their classes, and left early from class; total hours of standing per week were recorded.

Measurement of the metabolic equivalents (METs) of sitting and standing

The participants were required to attend a 1-hour metabolic testing session to measure the METs of sitting and standing. Because numerous factors affect the true resting metabolic rate, the participants were required to refrain from eating for 2-4 hours before the test, from consuming caffeine for at least 4 hours before the test, and from exercising. The test was rescheduled if the participant felt ill. The sessions occurred in this order: 1) seated at a desk for 20 minutes and 2) standing at a standing desk for 20 minutes with a 5 -minute break between each session. During each 20-minute session, the participant was asked not to talk or laugh. The participants could read, write, text, or watch videos if the activity was relaxing. At the start of each 20 -minute session, the participants were instructed to wear a mask apparatus with a breathing valve to collect expired gases. Expired gases were collected using the Parvo Medics TrueOne 2400 Metabolic Measurement System (Sandy, UT, USA). For analyses, the METs of the last 5 minutes of each sitting and standing session were averaged and recorded. A visual depiction of the protocol for the measurement of standing METs is shown in Figure 2.

\section{Statistical analysis}

All analyses were performed using SPSS Version 25.0 (IBM Corporation, Armonk, NY, USA). All variables were initially checked for normality using the KolmogorovSmirnov test. Measures of centrality and spread are presented as mean \pm SD. Mean cardiometabolic measures representative of the 3 weeks of sitting and 3 weeks of standing were calculated using the data collected from the weekly assessments. Next, MetS $z$-scores representative of the 3-week blocks of sitting and standing were determined. Differences in cardiometabolic factors and MetS $z$-scores between standing and sitting were analyzed with 


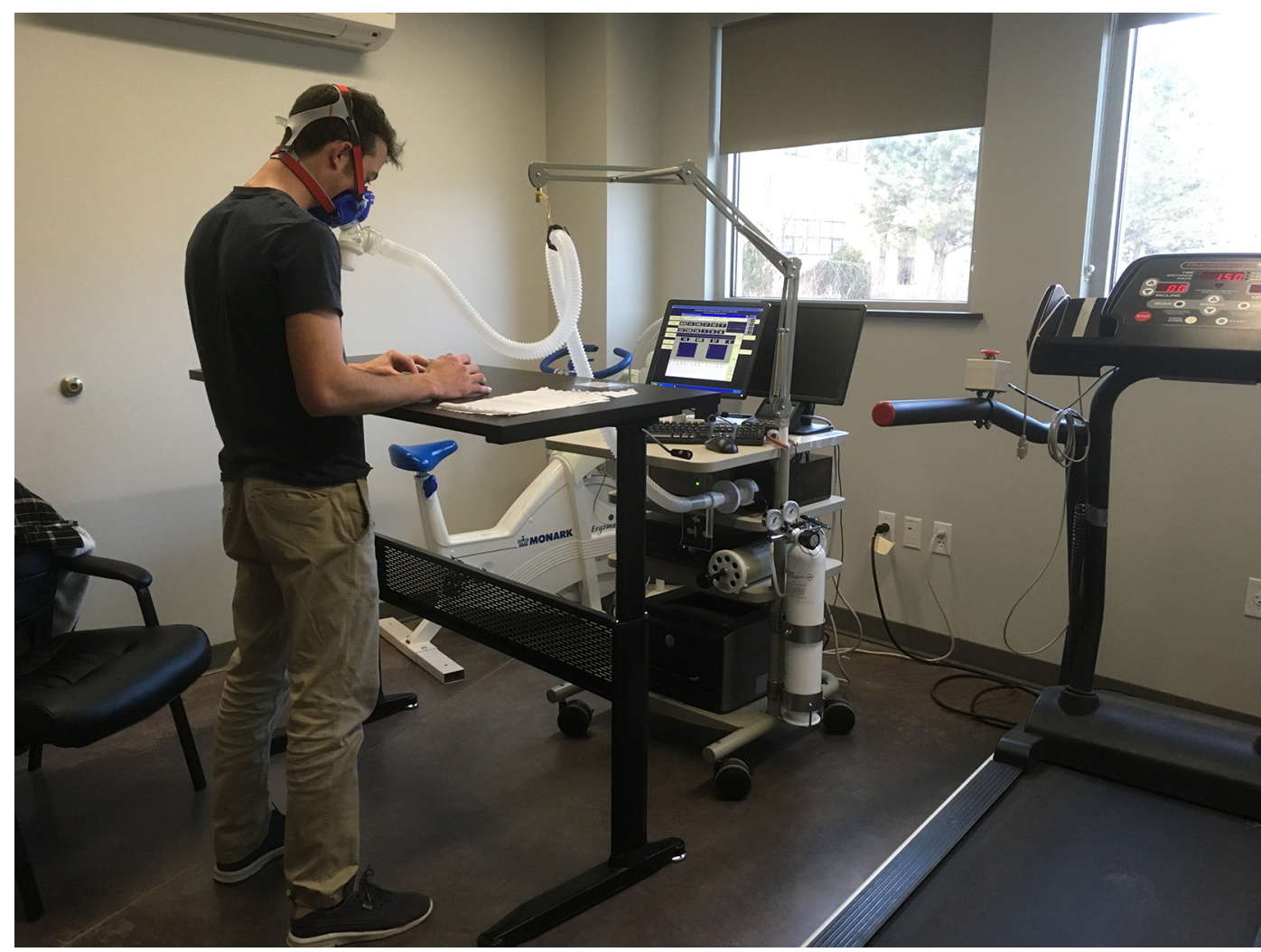

Figure 2 A study participant with the attached Parvo Medics metabolic analyzer during the measurement of standing METs. Abbreviation: METs, metabolic equivalents.

paired $t$-tests. Likewise, the difference in METs between standing and sitting was analyzed with a paired $t$-test. Lastly, the mean difference in MetS $z$-score between sitting and standing was calculated. To determine individual MetS $z$-score responsiveness to standing, delta values $(\Delta)$ were calculated (standing minus sitting) to establish the change $(\Delta)$ in MetS $z$-score. Subsequently, the participants were categorized as a "responder" if $\Delta$ was $<0$ or "nonresponder" if $\Delta$ was $>0$. The alpha level of statistical significance was set at $P<0.05$ for all analyses.

\section{Results}

All analyses and data presented in the results are for those participants $(n=21)$ who completed the intervention. One participant dropped out of the study because of a medical leave of absence from school not associated with the study. All participants acted as their own control in this crossover-design study. The participants were randomly assigned to sit or stand for 3 weeks followed by a 1-week (sitting) washout and 3 weeks of the opposite condition to the first 3 weeks. Both phases were well tolerated by all participants.

\section{Lifestyle}

As illustrated in Table 2, the sample on average exercised greater than the minimum recommendations by the American College of Sports Medicine. ${ }^{5}$

\section{Cardiometabolic risk factors and METs}

The mean measurements for cardiometabolic risk factors for 3 weeks of sitting and 3 weeks of standing along with METs are presented in Table 3. Paired $t$-tests revealed significant differences $(P<0.05)$ in all cardiometabolic risk factors between the 3 weeks of sitting and 3 weeks of standing time blocks. Moreover, MetS $z$-score was significantly improved $(P<0.05)$ during the 3 weeks of standing vs 3 weeks of sitting. The METs were significantly higher $(P<0.05)$ during standing than during sitting.

\section{Interindividual variability in MetS z-score responses to standing}

The incidence of MetS $z$-score responders and nonresponders to standing in the classroom when compared to sitting is presented in Figure 3. Although there was considerable 
Table 2 Physical activity and sedentary behavior (mean \pm SD) based on the International Physical Activity Questionnaire and Sedentary Behavior Questionnaire

\begin{tabular}{|l|l|l|l|}
\hline Parameter & Combined $(\mathbf{n}=\mathbf{2} \mathbf{I})$ & Males $(\mathbf{n}=\mathbf{I 3})$ & Females $(\mathbf{n}=\mathbf{8})$ \\
\hline IPAQ number of days with vigorous physical activity & $3.4 \pm 2.4$ & $3.0 \pm 2.6$ & $4.1 \pm 2.2$ \\
IPAQ time spent doing vigorous physical activity (minutes) & $80.5 \pm 83.8$ & $86.2 \pm 103.6$ & $71.3 \pm 39.2$ \\
IPAQ number of days with moderate physical activity & $4.2 \pm 2.3$ & $3.9 \pm 2.5$ & $4.6 \pm I .9$ \\
IPAQ time spent doing moderate physical activity (minutes) & $96.4 \pm 67.6$ & $92.3 \pm 66.6$ & $103.1 \pm 73.1$ \\
IPAQ number of days with walking & $4.4 \pm 2.4$ & $4.0 \pm 2.5$ & $5.1 \pm 2.0$ \\
IPAQ time spent walking (minutes) & $56.2 \pm 101.7$ & $67.8 \pm 127.1$ & $37.5 \pm 34.4$ \\
SBQ weekday sedentary behavior (minutes) & $44 I .4 \pm I 79.8$ & $469.6 \pm 205.6$ & $395.6 \pm I 26.5$ \\
SBQ weekend sedentary behavior (minutes) & $525.7 \pm 188.5$ & $576.9 \pm 202.8$ & $442.5 \pm I 35.1$ \\
\hline
\end{tabular}

Abbreviations: IPAQ, International Physical Activity Questionnaire; SBQ, Sedentary Behavior Questionnaire.

Table 3 Cardiometabolic and metabolic variables for sitting and standing (mean \pm SD) and mean differences (95\% Cl) between postures

\begin{tabular}{|l|l|l|l|}
\hline Parameter & Sitting $(\mathbf{n = 2 I})$ & Standing $(\mathbf{n = 2 I})$ & Mean difference $(\mathbf{9 5 \%}$ Cl) \\
\hline SBP $(\mathrm{mmHg})$ & $120.3 \pm 10.4$ & $117.4 \pm 9.5^{\mathrm{a}}$ & $-2.86(-0.69$ to -5.02$)$ \\
DBP $(\mathrm{mmHg})$ & $75.4 \pm 6.8$ & $74.0 \pm 6.9^{\mathrm{a}}$ & $-1.43(-0.7 \mathrm{I}$ to -2.15$)$ \\
Triglycerides $(\mathrm{mg} / \mathrm{dL})$ & $85.2 \pm 28.3$ & $75.4 \pm 22.1^{\mathrm{a}}$ & $-9.82(-4.69$ to -14.96$)$ \\
HDL cholesterol $(\mathrm{mg} / \mathrm{dL})$ & $54.8 \pm 14.4$ & $57.5 \pm 14.9^{\mathrm{a}}$ & $2.67(2.01-3.32)$ \\
Blood glucose $(\mathrm{mg} / \mathrm{dL})$ & $86.3 \pm 5.4$ & $84.5 \pm 5.6^{\mathrm{a}}$ & $-1.8 \mathrm{I}(-0.92$ to -2.70$)$ \\
METs & $1.02 \pm 0.07$ & $1.47 \pm 0.09^{\mathrm{a}}$ & $0.44(0.40-0.49)$ \\
MetS z-score & $-5.25 \pm 2.69$ & $-5.91 \pm 2.70^{\mathrm{a}}$ & $-0.66(-0.53$ to -0.78$)$ \\
\hline
\end{tabular}



Abbreviations: DBP, diastolic blood pressure; HDL, high-density lipoprotein; METs, metabolic equivalents; MetS, metabolic syndrome; SBP, systolic blood pressure.

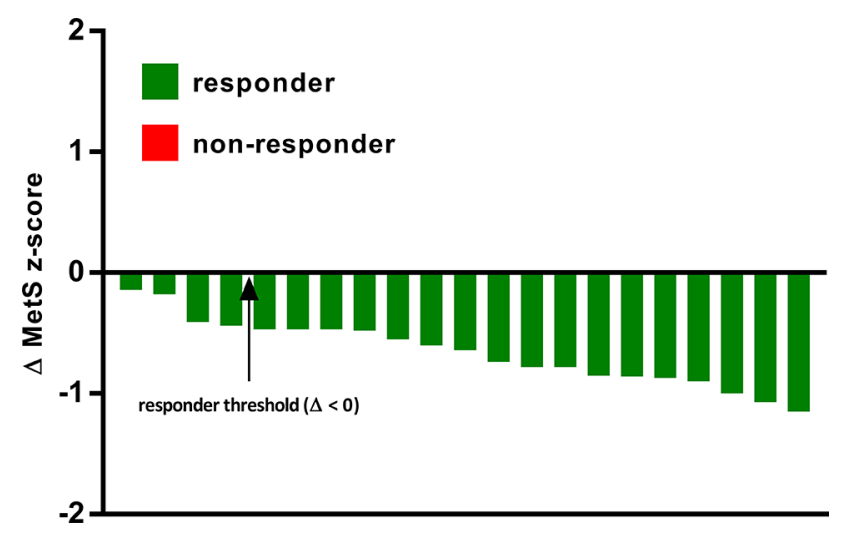

Figure 3 Interindividual variability in $\Delta$ MetS z-score responses to standing when compared to sitting.

Abbreviation: MetS, metabolic syndrome.

interindividual variability in the $\Delta$ MetS $z$-score response, there was a $100 \%(21 / 21)$ incidence of a favorable change (ie, responders) in MetS $z$-score response.

\section{Discussion}

The main finding of the present study was that a standing desk in the classroom paradigm significantly improved cardiometabolic health in a cohort of university students throughout a short 3 weeks time span. Overall, the intervention was well tolerated and therefore may be a promising modality to improve the cardiometabolic health profile of students who engage in high levels of sedentary behavior. Increasing standing time in the classroom, and therefore lessening weekly sedentary behavior, could be a potential, wide-scale, effective strategy for primordial prevention of cardiometabolic diseases.

\section{The active couch potato lifestyle}

In recent years, the term "active couch potato" has been used to describe people who meet PA recommendations yet spend the remainder of their waking hours engaged in primarily sedentary behaviors. Indeed, Table 2 indicates that the participants in the present study likely could be categorized as "active couch potatoes". It has been demonstrated that a negative dose-response relationship exists between sedentary behavior and waist circumference, blood pressure, and blood glucose levels, further emphasizing that sedentary behavior is a risk factor independent from physical inactivity. ${ }^{8}$ Moreover, the amount of time spent in sedentary behaviors is positively correlated with all-cause 
mortality, type 2 diabetes, cancer, high body mass index, CVD, and MetS. ${ }^{9}$ Therefore, for participants in the current sample and others in the population who spend the majority of their waking hours sedentary, exercising for an hour may not be enough to stave off the deleterious cardiometabolic effects from prolonged sitting.

\section{Cardiometabolic risk factors}

The relationship between sedentary behavior and cardiometabolic disease risk is well established..$^{8,10,11}$ From the findings of the present study and those of other studies, adjusting the physical environment of schools and offices can have a major impact with regard to health outcomes in students and workers alike. As more and more time per day is dedicated to low MET activities ( $\leq 1.5$ METs), in the name of productivity and/or entertainment, increasing standing time may be a simple, yet effective way to decrease the negative cumulative health consequences of prolonged sitting. To date, the present study is the first to assess the effects of increasing standing time on cardiometabolic risk factors in college students. Other studies on college students in this topic have focused on $\operatorname{cognition}^{12}$ and energy expenditure ${ }^{13}$ while at active workstations. As these studies differ in methodology and intention, it is difficult to relate them to the present study. However, on the basis of previous research on office workers, together with the present study, we can extrapolate and derive some conclusions on the efficacy of incorporating standing desks into the school/work environment. In this study, the participants were relatively healthy (Table 3 ), and yet, they experienced widespread subtle improvements in cardiometabolic health with reduced sitting. It may be surmised that the changes seen in a less healthy cohort would be greater. Indeed, several authors have reported that a standing intervention elicits improvements in various cardiometabolic risk factors such as HDL cholesterol, ${ }^{14}$ total cholesterol, and DBP. ${ }^{15}$ With regard to energy expenditure and substrate utilization, Gao et $\mathrm{al}^{10}$ found a significant increase in muscle activity (49.4\%), energy expenditure (9.2\%), and fat oxidation (fat usage increased from $39.4 \%$ to $48.3 \%$ and carbohydrate usage decreased from $60.6 \%$ to $51.7 \%$ ) when standing compared to that when sitting in 18 Finnish, female, middle-aged office workers (49.4 \pm 7.9 years). The increase in fat oxidation is vital as it may lead to improved insulin sensitivity over time, which is a primary goal when targeting reduced cardiometabolic disease risk. Additionally, increasing muscle activity and energy expenditure with each day, week, and year may result in gradual and sustained long-term improvements in one's lifetime cardiometabolic profile.

\section{Primordial prevention}

To take a step in the other direction, with regard to primordial prevention, as mentioned previously, the present study group was reasonably healthy and they still saw a positive change. This raises the question - are we too late in looking at college students? What would the cardiometabolic profiles of college students look like had they been standing in class since kindergarten? The current trend in primary education is to provide different "workstation" options for children, where they can choose to sit or stand. As children progress, however, through secondary school and then into college, the norm is to keep people in their seats. Therefore, by the time one enters the workplace, these habits are well formed and the road to poor health has been set in motion. The good news is that, on the basis of the modest yet significant changes seen in this study with 3 weeks of standing in the classroom, regardless of when sitting behavior changes during the lifespan, cardiometabolic risk factors may be reduced, reversed, or even prevented all together.

\section{Cardiometabolic outcomes: standing vs exercise training}

Interestingly, positive modifications in the cardiometabolic profile observed in the present study that occurred over the course of 3 weeks of classroom standing (Table 3 ) are comparable to modifications reported in the literature with regular aerobic exercise training. For example, the chronic benefits from aerobic exercise training in terms of blood pressure reduction are a decrease of 3 and $2 \mathrm{mmHg}$ in SBP and DBP, respectively, after anywhere between 1 and 6 months of training. ${ }^{16}$ Although these changes appear rather unassuming, it has been demonstrated that a blood pressure decrease of as little as $2 \mathrm{mmHg}$ is associated with a $6 \%$ decrease in stroke mortality and a $4 \%$ decrease in coronary artery disease. ${ }^{17}$ Three months of aerobic exercise training has been linked to increase in HDL cholesterol of 2-8 mg/ dL. ${ }^{18}$ This positive modification of the lipid profile yields important overall health benefits as it has been estimated that for every $1 \mathrm{mg} / \mathrm{dL}$ increase in HDL cholesterol, the risk of a coronary heart disease event is reduced by $2 \%-3 \% .{ }^{19}$ Triglycerides can also be attenuated following several months of regular exercise training with typical reductions between 5 and $38 \mathrm{mg} / \mathrm{dL} .{ }^{18}$ 


\section{Methodological considerations}

Thus far, the research design of studies on increasing standing time in office and other settings has been inconsistent in controlling for standing time as well as length of intervention. Despite methodological differences across studies, an emerging theme is that decreasing sitting time (and concomitantly increasing standing time) appears to be beneficial in improving cardiometabolic risk factors and increasing energy expenditure. To fully understand the impact of changing the work environment to include more standing time, it is imperative to have consistency with regard to the number of hours per day spent standing. Additionally, understanding the time component, with regard to weeks of standing, to elicit positive change in the cardiometabolic profile of sedentary adults is also needed.

\section{Conclusion}

The relationship between sedentary behavior and cardiometabolic disease risk is well established. As such, the results of the current study provide encouraging preliminary evidence that subtly adjusting the classroom environment to promote increased standing time and decreased sitting time can help improve cardiometabolic health of students in the university setting. A standing desk in the classroom paradigm could be a potential, wide-scale, effective strategy for primordial prevention of cardiometabolic diseases.

\section{Acknowledgment}

This study was supported by a grant from the American Council on Exercise.

\section{Disclosure}

The authors report no conflicts of interest in this work.

\section{References}

1. Schilter JM, Dalleck LC. Fitness and fatness: indicators of metabolic syndrome and cardiovascular disease risk factors in college students? J Exerc Physiol Online. 2010;13:29-39.

2. Dalleck LC, Kjelland EM. The prevalence of metabolic syndrome and metabolic syndrome risk factors in college-aged students. Am J Health Promot. 2012;27(1):37-42.
3. Yates T, Wilmot EG, Khunti K, Biddle S, Gorely T, Davies MJ. Stand up for your health: Is it time to rethink the physical activity paradigm? Diabetes Res Clin Pract. 2011;93(2):292-294.

4. Benzo RM, Gremaud AL, Jerome M, Carr LJ. Learning to stand: the acceptability and feasibility of introducing standing desks into college classrooms. Int J Environ Res Public Health. 2016;13(8):823.

5. Riebe D, Ehrman JK, Liguori G, Magal M, editors. ACSM's Guidelines for Exercise Testing and Prescription. 10th ed. Baltimore, MD: Lippincott Williams \& Wilkins; 2018.

6. Ramos JS, Dalleck LC, Borrani F, et al. Low-volume high-intensity interval training is sufficient to ameliorate the severity of metabolic syndrome. Metab Syndr Relat Disord. 2017;15(7):319-328.

7. Malin SK, Nightingale J, Choi SE, Chipkin SR, Braun B. Metformin modifies the exercise training effects on risk factors for cardiovascular disease in impaired glucose tolerant adults. Obesity (Silver Spring). 2013;21(1):93-100.

8. Maddison R, Jiang Y, Foley L, Scragg R, Direito A, Olds T. The association between the activity profile and cardiovascular risk. J Sci Med Sport. 2016;19(8):605-610.

9. Lee PH, Wong FK. The association between time spent in sedentary behaviors and blood pressure: a systematic review and meta-analysis. Sports Med. 2015;45(6):867-880.

10. Gao Y, Silvennoinen M, Pesola AJ, Kainulainen H, Cronin NJ, Finni T. Acute metabolic response, energy expenditure, and EMG activity in sitting and standing. Med Sci Sports Exerc. 2017;49(9):1927-1934.

11. Macewen BT, Saunders TJ, Macdonald DJ, Burr JF. Sit-stand desks to reduce workplace sitting time in office workers with abdominal obesity: a randomized controlled trial. J Phys Act Health. 2017;14(9):710-715.

12. Bantoft C, Summers MJ, Tranent PJ, Palmer MA, Cooley PD, Pedersen SJ. Effect of Standing or Walking at a Workstation on Cognitive Function: A Randomized Counterbalanced Trial. Hum Factors. 2016;58(1):140-149.

13. Fountaine CJ, Johann J, Skalko C, Liguori GA. Metabolic and energy cost of sitting, standing, and a novel sitting/stepping protocol in recreationally active college students. Int J Exerc Sci. 2016;9(2):223-229.

14. Alkhajah TA, Reeves MM, Eakin EG, Winkler EA, Owen N, Healy GN. Sit-stand workstations: a pilot intervention to reduce office sitting time. Am J Prev Med. 2012;43(3):298-303.

15. Graves LEF, Murphy RC, Shepherd SO, Cabot J, Hopkins ND. Evaluation of sit-stand workstations in an office setting: a randomised controlled trial. BMC Public Health. 2015;15:1145.

16. Fagard RH. Exercise is good for your blood pressure: effects of endurance training and resistance training. Clin Exp Pharmacol Physiol. 2006;33(9):853-856.

17. Chobanian AV Bakris GL, Black HR, et al; National Heart, Lung, and Blood Institute Joint National Committee on Prevention, Detection, Evaluation, and Treatment of High Blood Pressure, National High Blood Pressure Education Program Coordinating Committee. The Seventh Report of the Joint National Committee on Prevention, Detection, Evaluation, and Treatment of High Blood Pressure: the JNC 7 report. JAMA. 2003;289(19):2560-2572.

18. Durstine JL, Grandjean PW, Davis PG, Ferguson MA, Alderson NL, Dubose KD. Blood lipid and lipoprotein adaptations to exercise: a quantitative analysis. Sports Med. 2001;31(15):1033-1062.

19. Pasternak RC, Grundy SM, Levy D, et al. Task force 3: Spectrum of risk factors for CHD. J Am Coll Cardiol. 1990;27:964-1047.
Diabetes, Metabolic Syndrome and Obesity: Targets and Therapy is an international, peer-reviewed open-access journal committed to the rapid publication of the latest laboratory and clinical findings in the fields of diabetes, metabolic syndrome and obesity research. Original research, review, case reports, hypothesis formation, expert opinion and commentaries are all considered for publication. The manuscript management system is completely online and includes a very quick and fair peer-review system, which is all easy to use. Visit http://www.dovepress.com/testimonials.php to read real quotes from published authors. 